\title{
Inability of a Confocal Scanning Laser Doppler Flowmeter to Measure Choroidal Blood Flow in the Pig Eye
}

\author{
S. Pandav, W.H. Morgan, R. Townsend, S.J. Cringle and D.Y. Yu* \\ Centre for Ophthalmology and Visual Science, The University of Western Australia, Nedlands, Perth, Western Australia \\ 6009, Australia
}

\begin{abstract}
The aim of the study was to determine whether the Heidelberg Retinal Flowmeter (HRF), a confocal scanning laser Doppler flowmeter, can measure choroidal blood flow in pig eyes.

An HRF was used to obtain flow maps from in vitro pig eyes under a range of perfusion flow rates $(0-500 \mu \mathrm{L} / \mathrm{min})$ under conditions in which only the choroid was perfused. In some cases choroidal blood flow was also measured simultaneously using a conventional fiberoptic based Laser Doppler Perfusion Monitor (LDPM) which used the same laser wavelength $(780 \mathrm{~nm})$. The relationship between perfusion flow, HRF measured flow and LDPM measured flow was determined. HRF flow maps were also obtained in vivo as a function of focal plane setting through the retina and choroid.

Across the range of perfusion flow rates through the isolated eyes there was a poor correlation with HRF measured choroidal flow and perfusion flow. In contrast, there was a strong linear relationship between perfusion flow and LDPM measured blood flow. Both in vitro and in vivo, no choroidal vessels could be visualised in the HRF flow maps, even when the focal plane was in the choroid.

The HRF is unable to measure choroidal blood flow in pig eyes. This is not due to an inability of the $780 \mathrm{~nm}$ laser to penetrate into the choroid or due to red blood cell velocities in the choroid being higher than the measurement range of the instrument.
\end{abstract}

Keywords: Blood flow, choroid, hrf, heidelberg retinal flowmeter, laser doppler flowmetry.

\section{INTRODUCTION}

The ability to measure retinal and choroidal blood flow in a clinical environment has many applications in the diagnosis and monitoring of retinal diseases with an ischemic component. One of the commercially developed instruments for measuring retinal blood flow is the Heidelberg Retinal Flowmeter (HRF). This instrument uses a combination of Confocal Scanning Laser Ophthalmoscopy and Laser Doppler Flowmetry. It has been used to measure blood flow in retinal and optic disc blood vessels in a number of studies $[1,2]$. In addition, in a recent study in pigmented rats, the HRF was able to monitor blood flow in the major choroidal vessels [3]. In clinical studies it is generally accepted that the choroidal circulation makes a negligible contribution to the HRF measured flow signal. Exactly why the HRF instrument is unable to measure choroidal blood flow in a clinical situation is not known. An inability of the $780 \mathrm{~nm}$ laser to penetrate the RPE and return a large enough signal is one possibility. However, non scanning techniques using similar wavelengths have been used successfully to measure choroidal blood flow in man [4] and in animals [5]. Scanning techniques necessarily have a lower sampling rate than achievable with a stationary spot, but the advantage of a scanning technique is that a two dimensional map related to blood flow is obtained. In the case of the HRF instrument the

*Address correspondence to this author at the Centre for Ophthalmology and Visual Science, The University of Western Australia, Nedlands, Perth, Western Australia 6009, Australia; Tel: (618) 93810716;

Fax: (618) 93810700; E-mail: dyyu@cyllene.uwa.edu.au maximum sampling rate is $4 \mathrm{KHz}$, giving an upper Doppler bandwidth of $2 \mathrm{KHz}$. Given the relatively high blood flow rates in the choroid, an alternative explanation for the lack of a choroidal contribution to the HRF flow map in humans may be that the velocity of the red blood cells are outside the measurement range of the instrument. A better understanding of the apparent inability of the HRF device to image choroidal blood flow in man is required if improvements to the instrument in this respect are to be considered. Changes in choroidal blood flow are implicated in major retinal diseases such as macular degeneration, so an ability to generate two dimensional maps of choroidal blood flow would have immediate clinical applications. The present study seeks to assess the ability of the HRF device to monitor choroidal blood flow in the pig eye. We employed an isolated perfused pig eye technique in which blood flow could be manipulated over a very wide range, thereby producing a range of red blood cell velocities, some of which would certainly be within the upper range of the instrument. A direct comparison was also made between the HRF instrument and a non scanning blood flow measurement instrument employing the same laser wavelength. Some components of the study were also repeated in vivo in pigs to mimic an operating environment closer to that pertaining in a clinical setting.

\section{MATERIALS AND METHODOLOGY}

This study included in vitro as well as in vivo experiments on pig eyes. All experiments were performed at the Physiology and Pharmacology Centre, Centre for Ophthalmology and Visual Science, The University of Western Aus- 
tralia. All procedures involving animals were cleared by the Animal Ethics Committee of the University of Western Australia.

\section{Preparation of Blood}

Heparinized pig blood was used in all in vitro experiments. The blood was centrifuged then the plasma and the buffy coat were discarded. The packed RBCs were transferred to a heparinised $30 \mathrm{ml}$ plastic syringe, which was then loaded on a syringe pump. Another $30 \mathrm{ml}$ plastic syringe containing phosphate buffered saline (PBS) was loaded on the same pump. RBCs and PBS were mixed, in a ratio of 1 : 1 , during subsequent perfusion studies.

\section{In Vitro Experiments}

Thirteen freshly enucleated pig eyes were used for in vitro experiments. The eyes were dissected under an operating microscope (Carl Zeiss, OPMI 1, Germany) to expose the ophthalmic, retinal and ciliary arteries. The eye was kept wrapped in wet gauze to keep it moist. The cannula was connected to the $50 \% / 50 \%$ RBC mixture from the syringe pump and the perfusate pressure was measured with a pressure transducer close to the entry point to the eye.

\section{Isolated Eyes Perfused via the Ophthalmic Artery}

In seven eyes, the ophthalmic artery (OA) was cannulated. HRF flow images were then obtained before and after ligation of the retinal circulation. This confirmed the ability of the HRF instrument to detect flow in the retinal vessels, and allowed the effect of altering the plane of focus to be visualised. For measurements of choroidal blood flow, following ligation of the retinal circulation, the HRF camera was initially focussed at the plane of the NFL and then flow maps were acquired at focal planes from -1.5 to -3.5 Diopters relative to the NFL. This process was repeated at the same location following stepwise changes in perfusion rates between 0 and $500 \mu \mathrm{L} / \mathrm{min}$.

\section{Isolated Eyes Perfused via a Ciliary Artery}

In the remaining six eyes, a cannula was passed into one of the ciliary arteries instead of the OA, so that only the choroid in the hemisphere under test was perfused. In addition, a 200-micron diameter LDPM optic probe was inserted into the eye through a small hole in the pars-plana. The tip of the probe was positioned close to the retinal surface in an area free of major retinal vessels overlying the area of choroid perfused by the cannulated ciliary artery. The HRF camera was aligned to view the same location and HRF flow maps taken at focal planes -1.5 to $-3.5 \mathrm{D}$ from that of the NFL. Both HRF and LDPM measurements were taken over a range of perfusion rates $(0-400 \mu \mathrm{L} / \mathrm{min})$. At any given time only one laser was switched on to prevent the possibility of interference between the two instruments.

\section{In Vivo Experiments}

Six young pigs weighing $25-30 \mathrm{~kg}$ were used. Pigs were sedated with an intra muscular injection of Zoletil (Tiletamine $250 \mathrm{mg}+$ Zolezepam $250 \mathrm{mg}$, Virbac (Australia) Pty. Ltd.) $25 \mathrm{mg} / \mathrm{kg}$ body weight, followed by endotracheal intubation. Anaesthesia was maintained by a mixture of nitrogen $(\sim 5 \mathrm{~L} / \mathrm{min})$, oxygen $(\sim 3 \mathrm{~L} / \mathrm{min})$ and halothane $(0.5-1 \%)$ administered through a ventilator (Ventimeter Controller,
Air-Shields, Inc. Hatbora, Pennsylvania, USA). Appropriate depth of anaesthesia was maintained by adjusting the halothane concentration. Care was taken to have a stable blood pressure and respiratory rate. Cannulae were placed in the femoral artery, the anterior chamber of the eye and in the lateral ventricle of the brain to measure blood pressure (BP), intraocular pressure (IOP) and cerebrospinal fluid pressure (CSFP) respectively. The femoral vein was cannulated for intravenous delivery of heparin (2500 to 3000 units, Pharmacia \& Upjohn, (Perth) Pty. Ltd. Bentley WA, Australia). The animal was placed on a specially designed table.

The left eye was used for all HRF flow measurements. The pupil was dilated with $1 \%$ mydriacyl (Tropicamide, Alcon, Australia) and $0.1 \%$ atropine eye drops. An eye ring was sutured to the conjunctiva at the limbus to keep the eye steady. A zero diopter contact lens was put on the cornea to keep the optics clear. Retinal blood flow measurements were made using the HRF camera which was mounted on a microscope stand for easy manoeuvrability. The camera was initially focused on the retinal NFL. After taking pictures at the NFL, the focal plane was moved towards vitreous by at least two 0.5 Diopter steps. Then the focal plane was shifted towards choroid in 0.25 to 0.5 diopters steps to a depth of -3.5 diopters. Three images were acquired at each focal plane. At the end of the experiment, the pigs were sacrificed with an anaesthetic overdose delivered intravenously.

\section{Flow Measurements}

Blood flow measurements were performed using the HRF and LDPM measuring instruments.

\section{HRF Imaging}

A Heidelberg Retinal Flowmeter (Heidelberg Engineering $\mathrm{GmbH}$, Heidelberg, Germany) was used for all HRF measurements. HRF measures blood flow by measuring the variations in the intensity of the backscattered light from the illuminated tissue. These intensity variations are caused by interference between the light scattered by stationary tissues and the light scattered by moving RBCs (frequency shifted light). The intensity variations are then fast Fourier transformed to get the power spectrum of the frequency shifted components. The instrument and its operation have been described in detail elsewhere [6]. Briefly, it has a 780-nm diode laser source, confocal optics and a photo-detector to measure backscattered light. It scans an area of $10^{\circ} \times 2.5^{\circ}$ with image resolution of $256 \times 64$ pixels. Each of the 64 horizontal lines is scanned 128 times with a repetition rate of $4 \mathrm{KHz}$. Acquired data consists of 128 measurements at each of 16,384 locations. The software (HRF software version 1.02-1) allows various hemodynamic parameters to be calculated in the user selected area within the computer generated flow map.

\section{LDPM Measurements}

A Laser Doppler Perfusion Monitor (Moor Instruments Inc. Devon, England), with a fiberoptic probe, was used for LDPM measurements. It uses a near infrared light at $780 \mathrm{~nm}$ wavelength. The depth of measurement is reported to be about $1 \mathrm{~mm}$ and the volume of tissue sampled is typically about $1 \mathrm{~mm}^{3}$ [7]. The range of detectable measurement velocities lies between 0.001 and $10 \mathrm{~mm} / \mathrm{sec}$. The fiberoptic probe was placed close to the retinal surface. The incident 
near infrared light is scattered by the moving RBCs and the static tissues resulting in a frequency broadened backscattered light due to the Doppler effect. The backscattered light is collected by an optical fibre and measured by a photodetector. The signal is then analysed to extract relative flow measurements.

\section{Data Analysis}

HRF Flow values were extracted from the selected locations using a square window of 10x10 pixels. While selecting locations, care was taken to place the analysis window over the capillary area avoiding any visible retinal blood vessels. In experiments where the LDPM was used, the analysis window was placed on the retinal area 'illuminated' by the LDPM. This made sure that LDPM and HRF recorded flow signals at the same location. In sequential images the measurement window was aligned at the same location by using a transparent template. The template was created by
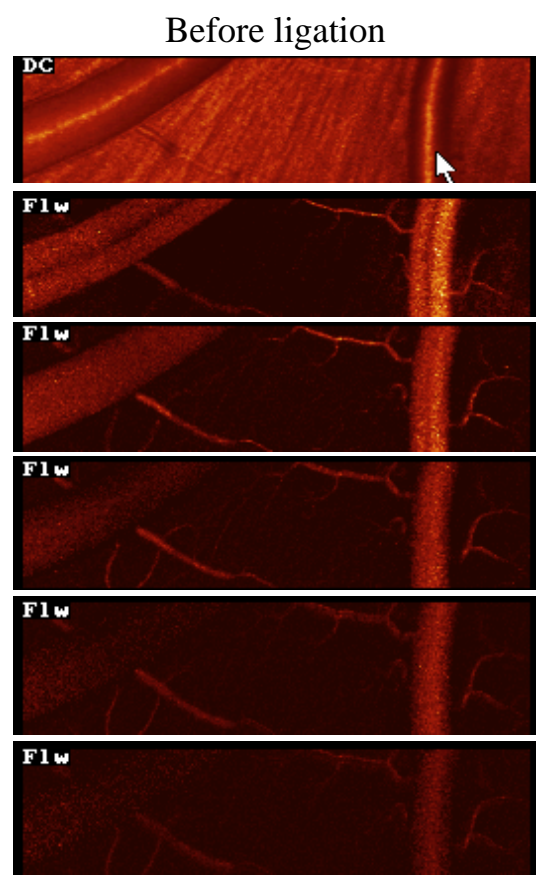

F1w
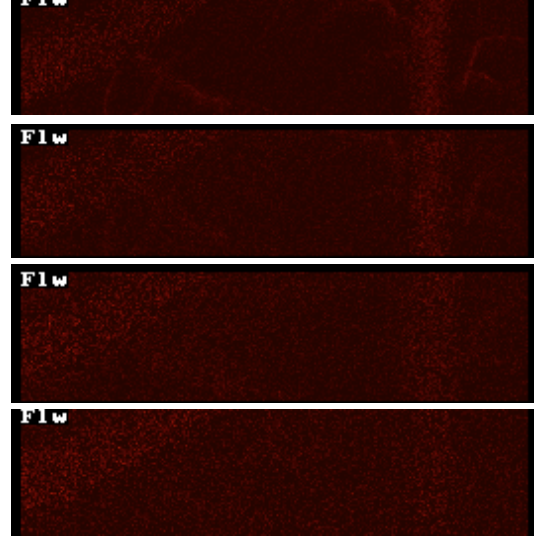

tracing the vessel outlines on an acetate film overlying the HRF flow image. In vivo experiments, three images were taken at each focal plane. The mean of the flow values acquired was used for subsequent analysis. In in vitro experiments, only one measurement was taken at each focal plane for a given flow rate. In both in vivo and in vitro studies the choroid was defined as being at tissue planes located $-2.5 \mathrm{D}$ $\pm 1 \mathrm{D}$ from the plane of the nerve fiber layer, and each of these locations was included in the choroidal average. Sigma Plot and Sigma Stat were used for data analysis (Systat Software, Point Richmond, CA). All values are expressed as mean \pm standard error. All error bars on graphs are standard errors.

\section{RESULTS}

\section{In Vitro Experiments}

Fig. (1) shows the typical appearance of flow maps before and after ligation of the retinal circulation. At a per-

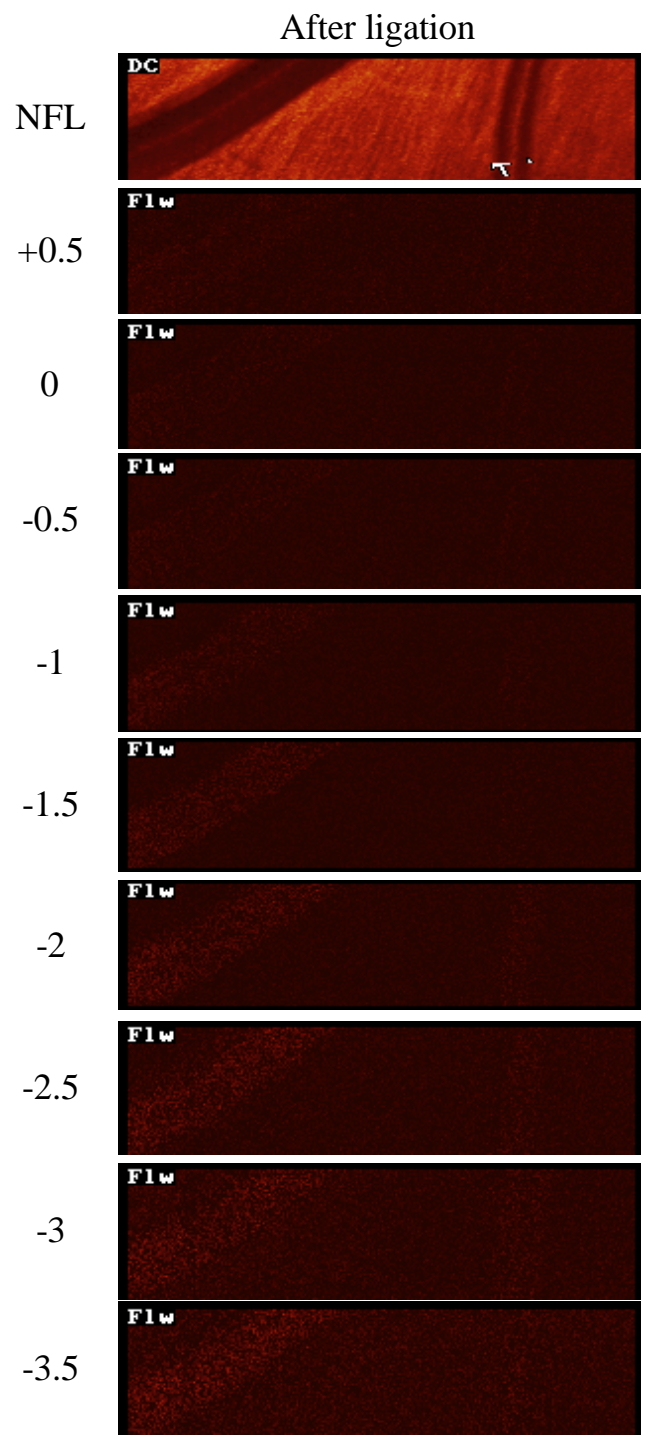

Fig. (1). HRF flow maps before and after ligation of retinal arteries. Ophthalmic Artery cannulated, perfusion rate $=500 \mu \mathrm{L} / \mathrm{min}$. Before ligation of the retinal arteries the retinal vasculature is clearly evident in the HRF flow maps when the focal plane is in the superficial retina (upper left panels). At deeper focal planes the retinal vasculature becomes less evident but there is still no pattern associated with the choroidal vessels in the HRF flow map. After ligation of the retinal arteries (right panels), the retinal vessels are no longer evident in the HRF flow map, and again there is no evidence of a choroidal flow pattern in the deeper focal planes. 
fusate flow rate of $500 \mu \mathrm{L} / \mathrm{min}$, the larger components of the retinal vasculature are well defined in the HRF flow map when the focal plane was set at the NFL. The flow signals from the retinal vessels become less distinct as the focal plane is moved towards and into the choroid, but no pattern of choroidal vessels is seen. After ligation of the retinal circulation no retinal vessels are visible at any focal plane, but again no choroidal vessels are seen, even when the focal plane is within the choroid.

Fig. (2) shows the HRF measured choroidal flow averaged for focal planes $-2.5 \mathrm{D} \pm 1 \mathrm{D}$ as a function of perfusate flow rate with the retinal circulation ligated. There is no significant relationship between HRF measured choroidal flow and perfusate flow rate.

HRF Measured Choroidal Flow with Retinal Artery Ligated

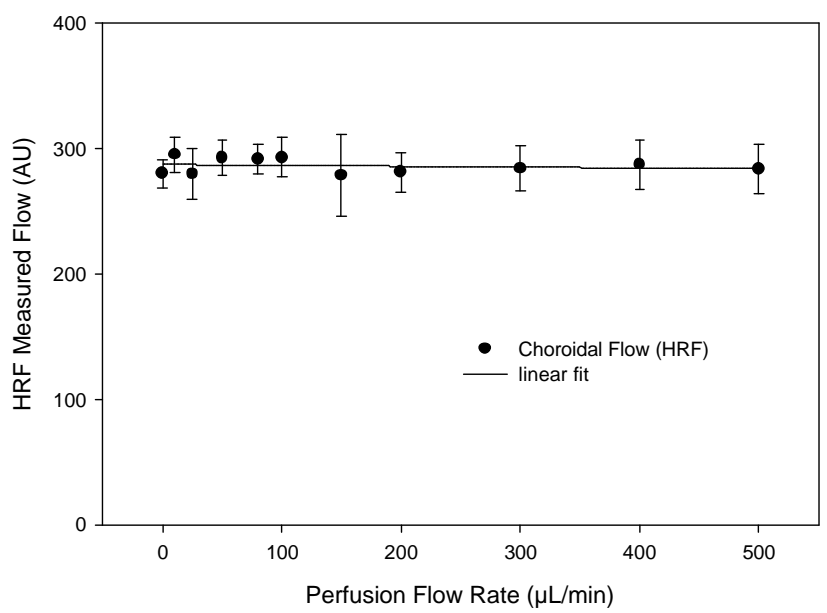

Fig. (2). HRF measured flow in the choroid, at different perfusion rates in a pig eyes perfused via the ophthalmic artery. There was no relationship between perfusion flow rate and HRF measured flow.

\section{LDPM Measured Flow Versus HRF Measured Flow in Pig Eyes Perfused via a Ciliary Artery}

In this set of experiments the choroid was perfused through one of the ciliary arteries. This negated the requirement to ligate the retinal vessels and also removed the possibility of a localised area of choroidal non perfusion due to vascular occlusion. With the ophthalmic artery cannulation the perfusate is delivered to every quadrant of the choroid, but with a ciliary artery cannulation the majority of the perfusate is delivered to the side of the globe under investigation. In addition, to ensure that the HRF imaged area of the choroid was indeed perfused, choroidal flow in the area under study was also monitored using the LDPM probe. Fig. (3) shows the LDPM probe in position and the HRF measurement window in the same region of retina at a perfusate flow rate of $400 \mu \mathrm{L} / \mathrm{min}$ through the ciliary artery. Non perfused retinal vessels can be seen in the DC image, but they are absent from the HRF flow map. At deeper focal planes there is no evidence of choroidal vessels in the HRF flow map.

The relationship between HRF measured flow and perfusion flow at the same location is shown in Fig. (4). There is no relationship between HRF measure flow and perfusion rate.

$\mathrm{DC}$
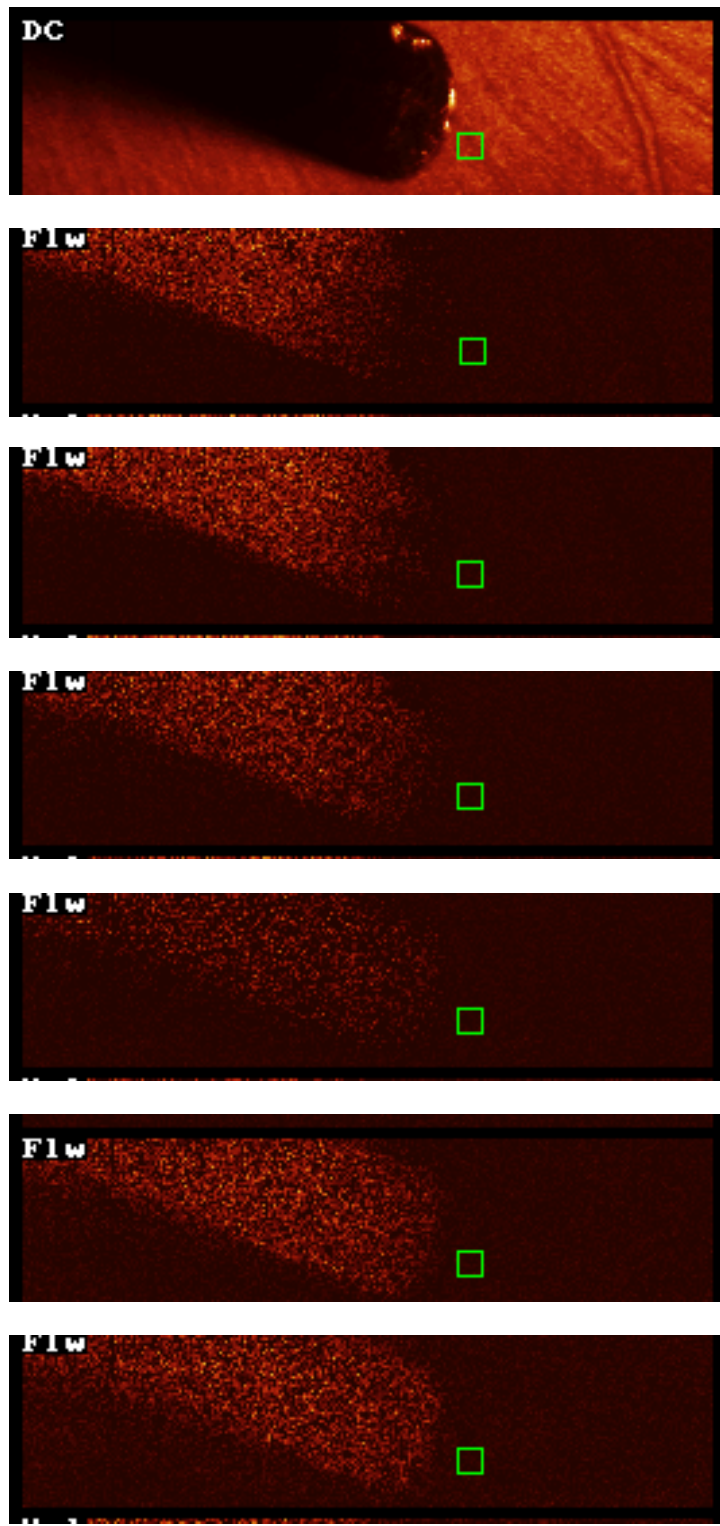

$-3$

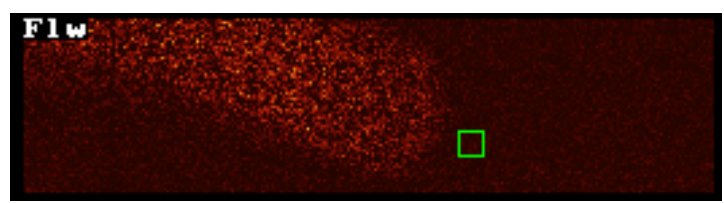

Fig. (3). LDPM probe in position adjacent to the area measured by the HRF (green box) in an eye in which the ciliary artery feeding this area of choroid was perfused. Note the absence of any retinal vessels in the HRF flow map and the absence of visible choroidal vessels.

Fig. (5) shows the averaged data for LDPM measured flow as a function of perfusate flow. The relationship is highly linear between perfusion flow and LDPM measured flow. 
HRF Measured Choroidal flow with ciliary artery cannulated

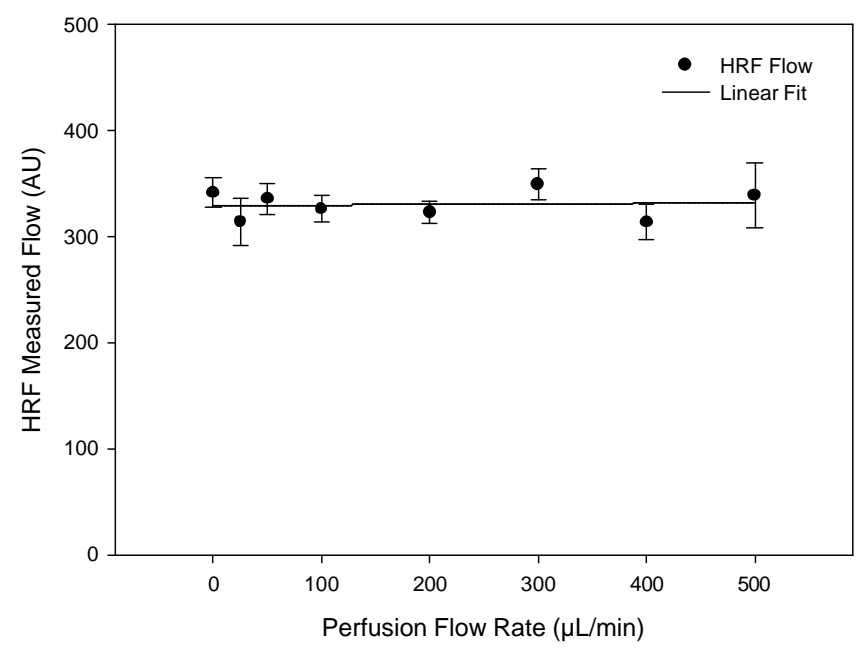

Fig. (4). HRF measured flow at different perfusion rates through the ciliary artery feeding the area of choroid being examined. HRF measure flow bears no relationship to perfusion flow.

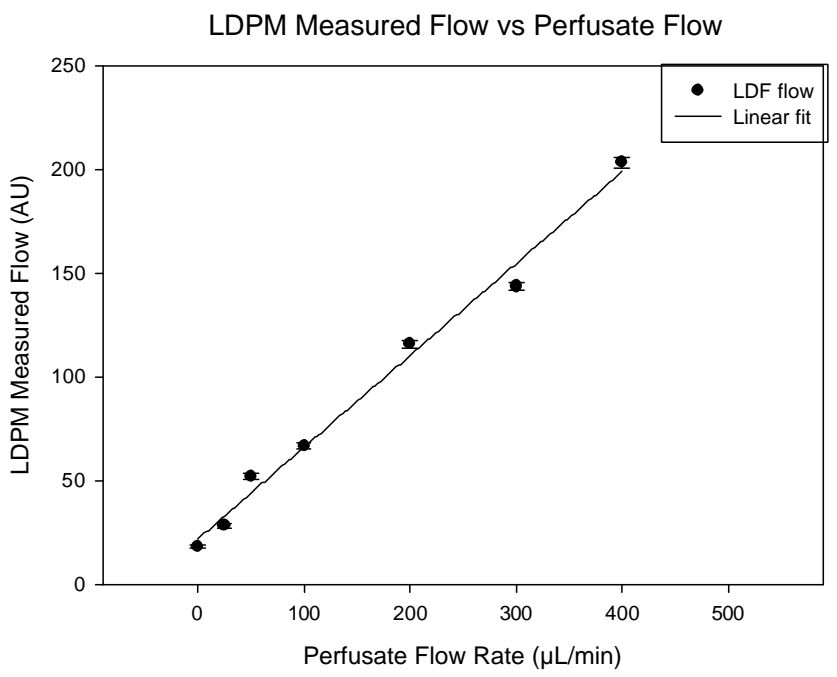

Fig. (5). LDPM measured flow at different perfusion rates under the same conditions as Fig. (4). A highly linear relationship exists between LDPM measure flow and perfusate flow rate. The LDPM device uses the same wavelength $(780 \mathrm{~nm})$ as the HRF instrument.

\section{In Vivo Experiments}

In vivo, $\mathrm{HRF}$ measurements at 8 locations in 6 pig eyes were recorded. Representative flow maps at different focal planes are shown in Fig. (6). Retinal vascular details were well defined when the focus was at the level of the NFL but became blurred as the focal plane moved deeper (from 0 to $-3.5 \mathrm{D})$. No choroidal vessels were visible at any focal depth. The average HRF measured flow in the choroid was $252.0 \pm 20.1$ AU.
DC

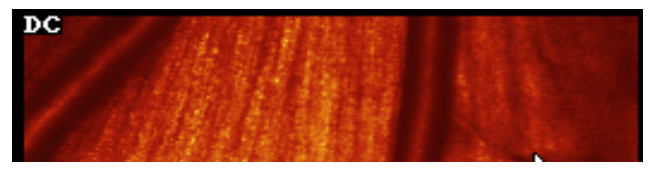

$+0.5$

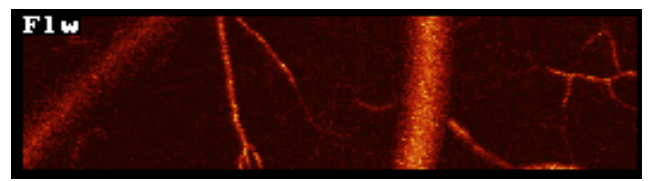

NFL
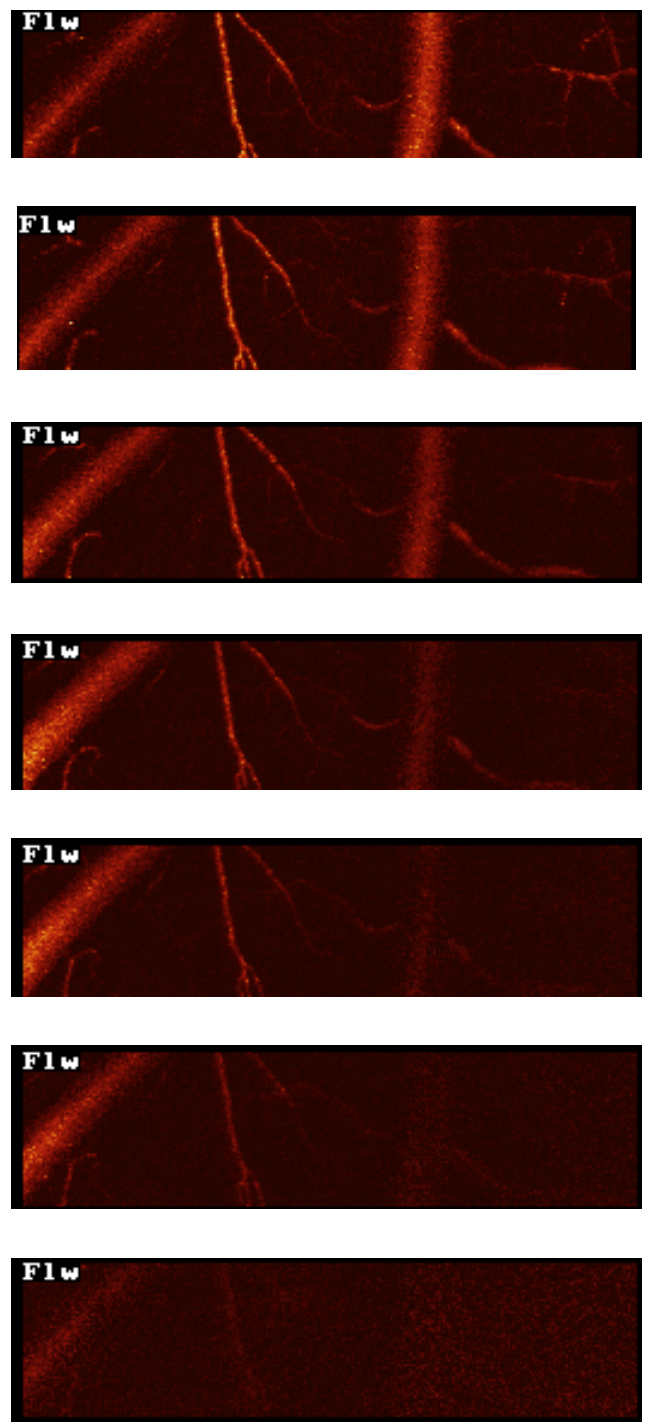

Fig. (6). HRF flow maps, from an in vivo experiment, taken at different focal planes. The retinal vessels are clearly visible when the focal plane is in the superficial retina. At deeper focal planes the retinal vessels are less evident but choroidal vessels are not seen.

\section{DISCUSSION}

A clinically usable method of mapping choroidal blood flow would be of great value. In recent years there has been a lot of interest in measuring choroidal blood flow in healthy eyes as well as eyes with various disease conditions $[4,8,9]$. The Heidelberg Retinal Flowmeter (HRF) is a commercially 
available instrument widely used for generating perfusion maps of the retinal and optic disk vasculature $[1,2,7,10,11]$. It is generally accepted that the HRF instrument does not detect choroidal flow in routine clinical use. Why choroidal flow is not detected is not well understood. From a simplistic viewpoint it seems surprising that blood flowing in the choroidal vasculature does not swamp the HRF flow signal produced by the retinal circulation. The choroid is highly vascular, and the capillaries form an almost continuous sheet of fast flowing blood. Some estimates put the rate of choroidal blood flow to be 25 times that of the retinal circulation [12]. The absence of a choroidal signal in HRF flow maps deserves further investigation in the hope that a modified noninvasive technique can be developed which is able to generate perfusion maps of the choroidal circulation in a clinical environment.

The present study demonstrates that the conventional HRF instrument is not able to measure choroidal blood flow in the pig eye. This is also true under low flow conditions in which the velocity of red blood cells is anticipated to produce Doppler frequency shifts less than the upper frequency limit of the instrument. In an isolated eye perfused via the ophthalmic artery it could be argued that there is a possibility that localised areas of non-perfusion may be present due to localised vascular occlusion. Given the highly vascular nature of the choroid and the relatively wide bore of all choroidal vessels, including the choriocapillaris, the prospect of local vascular occlusion seems remote. However, to address this issue we also performed studies in pig eyes perfused via a ciliary artery. This lessens the likelihood of local nonperfusion as only one half of the eye is being perfused, offering fewer alternative routes for perfusate flow. In addition, we also monitored choroidal flow in the same area using a non-scanning laser perfusion monitor to directly confirm the presence of choroidal perfusion in the area in which the HRF measurement was performed. Finally we were able to demonstrate that choroidal vessels could not be visualised in the HRF flow maps obtained from in vivo pigs where any issues related to abnormal tissue perfusion are eliminated.

It is important to investigate why choroidal vessels do not show up in the HRF flow maps. We have ruled out the possibility that perfusion rates are too high, since we see no choroidal vessels at perfusion rates close to zero. We have also shown that an alternative laser Doppler flowmetry device, using the same wavelength, has a linear response to choroidal flow. Thus an inability of $780 \mathrm{~nm}$ laser light to penetrate the RPE cannot be the sole explanation. A more likely explanation is that the effective sensitivity of the two instruments is different. The LDPM device measures the total light received from a fixed volume of tissue, whereas the HRF device is sampling light from each pixel of interest for only a small fraction of the time required for each scan. The confocal nature of the HRF, achieved by pinhole optics, must also necessarily reduce the Doppler shifted light being detected. Despite the confocal nature of the instrument it must be remembered that flow signals are often summed from a considerable retinal depth. It has been noted in thinner areas of retina, such as in the avascular region of the rabbit retina, choroidal vessels can be visualised in the HRF flow map, even when the focal plane is set to the retinal surface [13]. This however was not true of the thicker vascularised portion of the rabbit retina in that study. Retinal thickness may therefore also play a part in determining the ability to identify choroidal vessels in HRF flow maps. Retinal thickness cannot be the only factor since, in pigmented rats, which have a retinal thickness of $\sim 300-350 \mu \mathrm{m}$ (slightly thicker than human or pig retina), the major choroidal vessels were clearly visualised in HRF flow maps [3]. Factors such as tissue reflectance, scattering properties, and the influence of the structural properties of RPE/Bruch's membrane also need to be considered.

\section{CONCLUSION}

The existing HRF instrument is unable to measure choroidal blood flow in the pig eye. The fact that choroidal vessels have been visualised by the HRF in some animal studies suggests that it must be a combination of factors that determines the presence or absence of choroidal flow signals in the HRF flow maps. There appears to be scope for further modifications of the HRF instrument to allow choroidal perfusion maps to be generated in a clinical setting.

\section{ACKNOWLEDGEMENTS}

The authors wish to thank Dean Darcey for his invaluable assistance. We also wish to thank Heidelberg Engineering for the loan of the HRF instrument and for technical support.

Grant support was provided by the National Health and Medical Research Council of Australia and the Australian Research Council Centre of Excellence in Vision Science.

\section{REFERENCES}

[1] Hafez AS, Bizzaro RLG, Rivard M, Lesk MR. Changes in optic nerve head blood flow after therapeutic intraocular pressure reduction in glaucoma patients and ocular hypertensives. Ophthalmology 2003; 110(1): 201-10.

[2] Michelson G, Welzenbach J, Pal I, Harazny J. Functional imaging of the retinal microvasculature by scanning laser Doppler flowmetry. Int Ophthalmol 2001; 23(4-6): 327-35.

[3] Yu DY, Townsend R, Cringle SJ, Chauhan BC, Morgan WH. Improved interpretation of flow maps obtained by scanning laser Doppler flowmetry using a rat model of retinal artery occlusion. Invest Ophthalmol Vis Sci 2005; 46(1): 166-74.

[4] Riva CE, Cranstoun SD, Grunwald JE, Petrig BL. Choroidal blood flow in the foveal region of the human ocular fundus. Invest Ophthalmol Vis Sci 1994; 35(13): 4273-81.

[5] Kiel JW, Shepherd AP. Autoregulation of choroidal blood flow in the rabbit. Invest Ophthalmol Vis Sci 1992; 33(8): 2399-410.

[6] Michelson G, Schmauss B. Two dimensional mapping of the perfusion of the retina and optic nerve head. Br J Ophthalmol 1995; 79(12): 1126-32.

[7] Ludovico J, Bernardes R, Pires I, Figueira J, Lobo C, Cunha-Vaz J. Alterations of retinal capillary blood flow in preclinical retinopathy in subjects with type 2 diabetes. Graefes Arch Clin Exp Ophthalmol 2003; 241(3): 181-6.

[8] Schmetterer L, Strenn K, Findl O, et al. Effects of antiglaucoma drugs on ocular hemodynamics in healthy volunteers. Clin Pharmacol Ther 1997; 61(5): 583-95.

[9] Dimitrova G, Kato S, Tamaki Y, et al. Choroidal circulation in diabetic patients. Eye 2001; 15(Pt 5): 602-7.

[10] Hafez AS, Bizzarro RL, Lesk MR. Evaluation of optic nerve head and peripapillary retinal blood flow in glaucoma patients, ocular 
hypertensives, and normal subjects. Am J Ophthalmol 2003; 136(6): 1022-31.

[11] Chauhan BC, Smith FM. Confocal scanning laser doppler flowmetry: Experiments in a model flow system. J Glaucoma 1997; 6(4): $237-45$.
[12] Alm A, Bill A. Ocular Circulation. In: Hart WM, Moses RA, Eds. Adler's physiology of the eye: Clinical application. Mosby: St Louis, 1987: 183-99.

[13] Tamaki Y, Araie M, Fukaya Y, Ishi K. Validation of scanning laser Doppler flowmetry for retinal blood flow measurements in animal models. Curr Eye Res 2002; 24(5): 332-40.

(C) Pandav et al.; Licensee Bentham Open.

This is an open access article licensed under the terms of the Creative Commons Attribution Non-Commercial License (http: //creativecommons.org/licenses/by$\mathrm{nc} / 3.0 /$ ) which permits unrestricted, non-commercial use, distribution and reproduction in any medium, provided the work is properly cited. 